\title{
Construcción y validación de una escala de autoeficacia en el trabajo
}

\author{
María L. Campos-Carreño*, Camila B. Velasco, Javiera P. Araya y Carolina E. Gaete \\ Facultad de Humanidades, Departamento de Psicología, Universidad de La Serena, La Serena-Chile. \\ (Correo-e: mcampos@userena.cl; cvelasco@userena.cl; javiera.arayac@userena.cl; cgaete@userena.cl)
}

Recibido Oct. 21, 2020; Aceptado Dic. 16, 2020; Versión final Ene. 29, 2021, Publicado Jun. 2021

\begin{abstract}
Resumen
El objetivo de este estudio fue construir y validar una escala de autoeficacia en el trabajo aplicable a contextos laborales transversales. La muestra estuvo compuesta por 332 trabajadores chilenos divididos aleatoriamente en dos submuestras. El proceso de investigación se realizó en 4 etapas: 1) Diseño de instrumento, 2) validación de contenido, 3) análisis factorial exploratorio (submuestra A) y 4) análisis factorial confirmatorio (submuestra B). Los resultados para la escala total muestran indicadores de ajuste adecuados $(\mathrm{CFI}=0.97$; $\mathrm{TLI}=0.97$; RMSEA $=0.05$; alfa de Cronbach $=0.948$ ). La escala quedó constituida por 5 dimensiones: autogestión, transferencia, metacognición, meta-aprendizaje y autocuidado. Las alfas de Cronbach (0.858; $0.869 ; 0.808 ; 0.826$ y 0.854 respectivamente) certifican adecuada consistencia interna. Se concluye que la escala de autoeficacia en el trabajo generada presenta características psicométricas adecuadas para su uso, tanto a nivel de escala total como en cada una de sus dimensiones.
\end{abstract}

Palabras clave: autoeficacia; escala Likert; contexto laboral; psicología; trabajo; organizaciones

\section{Construction and validation of a self-efficacy scale at work}

\begin{abstract}
The main objective of this study was to construct and validate a scale of self-efficacy at work that is applicable to transversal work contexts. The sample consisted of 332 Chilean workers randomly divided into two subsamples. The study was conducted in four phases: 1) instrument design, 2) content validation, 3) exploratory factor analysis (subsample A), and 4) confirmatory factor analysis (subsample B). The results for the full scale showed adequate adjustment indicators $(\mathrm{CFI}=0.97 ; \mathrm{TLI}=0.97 ; \mathrm{RMSEA}=0.05$; Cronbach's alpha $=0.948)$. The scale was conformed into five dimensions: self-management, transfer, metacognition, meta-learning, and self-care. Their respective Cronbach's alphas $(0.858 ; 0.869 ; 0.808 ; 0.826$ and 0.854$)$ certified adequate internal consistency. In conclusion, the self-efficacy at work scale generated here showed adequate psychometric characteristics for its use, both at the level of the entire scale and at level of each of its dimensions.
\end{abstract}

Keywords: self-efficacy; Likert scale; workplace; work; organizational psychology 


\section{INTRODUCCIÓN}

La autoeficacia constituye uno de los constructos de mayor relevancia para responder a las demandas de bienestar, calidad, innovación y productividad de las organizaciones debido a su efecto directo en el comportamiento-desempeño laboral y a causa del impacto indirecto en los niveles de motivación, metas y aspiraciones, satisfacción laboral, tendencias afectivas y expectativas de resultados (Pepe et al., 2010; Calderón et al., 2017). La autoeficacia tiene sus orígenes en la Teoría Social Cognitiva y es definida como "las creencias en las propias capacidades para organizar y ejecutar los cursos de acción necesarios para la producción de determinados logros o resultados" (Bandura, 1997). Las creencias de autoeficacia son específicas a ciertos dominios y se desarrollan dependiendo de la actividad que se realice: escolar, social y profesional (Pepe et al., 2010) En consecuencia, el estudio de la autoeficacia en el trabajo resulta adecuado como contexto situado (Pepe et al., 2010).

El presente estudio define autoeficacia en el trabajo como las creencias que tiene cada trabajador respecto al conjunto de conocimientos, habilidades, actitudes y valores que le permiten concretar sus metas laborales, utilizando de manera óptima los recursos personales y contextuales disponibles en pos de desempeñar con calidad las tareas definidas. La versatilidad del concepto responde a las diversas dimensiones que lo estructuran destacando entre ellas: 1) la transferencia de los logros alcanzados (Criollo, Romero y Fontaines, 2017); 2) el autocuidado que permite mantener un bienestar biopsicoemocional lo que favorece la autoconfianza, esperanza en el futuro y confianza en el trabajo (Park, Song y Kim, 2020); 3) la autogestión que determina las decisiones que las personas realizan, el esfuerzo que despliegan y la perseverancia que sostienen frente a los desafíos (Bandura, 1997); 4) la metacognición que se relaciona con el proceso de análisis, planificación, acción de tareas y conciencia de metas (Colthorpe et al., 2019) y 5) el meta-aprendizaje que implica la capacidad de utilizar estrategias efectivas de aprendizaje lo que potencia la autorregulación de pensamientos, sentimientos y acciones favoreciendo la conquista de objetivos (Colthorpe et al., 2019).

Updegraff (2004) plantea la relevancia de dos conceptos en la autoeficacia: 1) la autoconciencia conceptualizada como el conocimiento de sí en cuanto a las capacidades y motivaciones que benefician tanto a sí mismo como a la organización en su conjunto y 2) la autodirección entendida como la conciencia del valor estratégico de la planificación, dando sentido a lo que se está haciendo en el aquí y ahora en función a metas específicas, medibles, alcanzables, realistas y oportunas.

El estudio de la autoeficacia es relevante por cuanto a mayor percepción de ésta mayor capacidad para hacer frente a las crisis y superar los fracasos favoreciendo la elección de tareas más complejas, compromisos con metas más altas, manejo efectivo de situaciones estresantes y recursos, mayor motivación, menos reacciones emocionales adversas, menores niveles de estrés, mayor bienestar laboral y afrontamiento activo de los problemas, mientras que una baja autoeficacia se asocia a depresión, ansiedad y desamparo (Bandura, 1997; Pepe et al., 2010; Calderón et al., 2017; Bargsted et al., 2019). Se destaca su rol mediador entre calidad laboral y salud (Bargsted et al., 2019) y entre competencias y rendimiento (Vera et al., 2011). Un alto nivel de autoeficacia predispone al trabajador a interpretar las exigencias del entorno como retos y no como sucesos incontrolables, ya que considera contar con las capacidades y habilidades suficientes para afrontar las demandas laborales manteniendo el foco en la tarea, siendo la autoeficacia un amortiguador de las exigencias ambientales lo que aporta al bienestar del individuo (Pepe et al., 2010; Bargsted et al., 2019).

Respecto a instrumentos de evaluación de autoeficacia, en Chile se encuentra la Escala de Autoeficacia General (EAG), desarrollada por Schwarzer y Jerusalem el año 1979, validada por Cid, Orellana y Barriga (2010), que mide el constructo a nivel general. A ella se suma una variedad de instrumentos orientados a medir la autoeficacia en el ámbito escolar/académico entre la que podemos mencionar a Ramos-Villagrasa et al. (2018) quienes adaptaron al español "Self-Efficacy for Writing Scale" (SEWS). Considerando la relevancia de disponer de instrumentos de medición de autoeficacia referidos al trabajo, la presente investigación tiene por objetivo construir y validar una escala de Autoeficacia en el Trabajo aplicable a contextos laborales transversales.

\section{MÉTODO}

La investigación corresponde a un diseño de tipo instrumental transversal (Montero y León, 2007), y se presenta en dos subsecciones: i) participantes; y ii) procedimiento y análisis de datos

\section{Participantes}

La muestra fue de tipo no probabilística y compuesta por 332 sujetos de la Región de Coquimbo, Chile pertenecientes a los siguientes sectores laborales: minería (supervisores y operadores), educación (directivos y docentes), salud (profesionales y técnicos), servicios administrativos y contables (profesionales y técnicos) y seguridad (guardias). Todos trabajadores activos con jornada laboral completa y contrato vigente al 
momento de realizar el estudio. La muestra fue aleatoriamente dividida en dos submuestras utilizando el programa SPSS v. 22. La primera de ellas (submuestra A) se compone de 173 sujetos con una edad media de 44.02 años (DT=9.8), de los cuales $33.5 \%$ eran hombres $(n=58)$ y $66.5 \%$ mujeres $(n=115)$. La muestra para la evaluación final de la escala (submuestra B) quedó conformada por 159 sujetos con edad media de 44.18 años $(D T=6.2)$, siendo $66.7 \%$ hombres $(n=106)$ y $33.3 \%$ mujeres $(n=53)$.

\section{Procedimiento y análisis de datos}

El estudio comprendió las siguientes fases: 1) Diseño de instrumento; 2) Validación de contenido; 3) Análisis factorial exploratorio y 4) Análisis factorial confirmatorio.

\section{Fase 1: Diseño de instrumento}

A partir de 4 grupos focales realizados con trabajadores de comercio, turismo, fruticultura y minería de la región de Coquimbo y expertos en psicología del trabajo y las organizaciones, se desarrolló el proceso de construcción de reactivos de tipo diferencial semántico en torno al constructo de autoeficacia en el trabajo. Se generaron 52 reactivos en base a un formato correspondiente a Escala Likert.

\section{Fase 2: Validación de contenido}

Los 52 ítems, establecidos por los grupos focales, fueron analizados por un grupo de expertos compuesto por miembros del Servicio Nacional de Capacitación y Empleo, Cámara de Comercio de Chile, Cámara Regional de Turismo, Corporación Regional de Desarrollo Productivo y psicólogos expertos en el área OrganizacionalLaboral, quienes establecieron las siguientes dimensiones en torno a las cuales organizaron los ítems: 1) Autogestión, entendida como la autovaloración de las capacidades de actuar por iniciativa propia, logrando desenvolverse estratégicamente en contexto para alcanzar metas, priorizando tareas y optimizando el uso del tiempo y los diversos recursos disponibles en la organización (Cerda, López, Osses y Saiz, 2015); 2) Transferencia, conceptualizada como la autovaloración de la capacidad de desplegar saberes adquiridos en diferentes contextos y aplicarlos en el ámbito laboral en torno al logro de un resultado (Gonczy, 2001); 3) Meta-cognición, comprendida como la autovaloración de la capacidad reflexiva que permite descifrar y reconocer elementos relevantes, anteponerse a eventos, construir hipótesis pertinentes, gestionar la variedad de tareas y tomar conciencia de fortalezas y debilidades al momento de realizar una tarea logrando la meta propuesta (McCluskey et al., 2013); 4) Meta-aprendizaje, definida como la autovaloración de la capacidad de tomar conciencia e identificar y desarrollar estrategias para lograr un aprendizaje efectivo, ejerciendo control sobre el proceso de avanzar en la zona de desarrollo próximo (Bandura, 1989) y 5) Autocuidado, entendida como la autovaloración de la capacidad de identificar y regular factores que afectan su bienestar personal, autorregulándose, accionando estrategias y recursos personales en pos de su bienestar y desempeño laboral (Cancio-Bello et al., 2020).

Los 52 ítems, distribuidos en las cinco dimensiones, fueron validados a nivel de contenido por tres jueces expertos en recursos humanos y lingüística. Estos jueces evaluaron los reactivos por medio de un formulario acorde a los siguientes criterios: correspondencia a la dimensión que pertenece cada ítem, ajuste a la intencionalidad semántica de cada reactivo, comprensión, redacción y habitualidad. El trabajo de estos jueces permitió establecer la escala inicial de Autoeficacia en el Trabajo constituida por 41 ítems.

\section{Fase 3: Análisis factorial exploratorio}

La versión de 41 ítems fue aplicada a la submuestra A por psicólogos previamente capacitados, quienes entregaron las instrucciones de manera oral. La aplicación se realizó grupalmente dentro de la jornada laboral de los participantes, con una duración de 20 minutos aproximadamente. Cada actor social respondió vía lápiz y papel de manera autónoma. Todos los participantes accedieron a ser parte de la investigación de forma voluntaria, lo cual fue corroborado mediante consentimientos informados individuales (i.e. participación voluntaria, consecuencias de la aplicación, garantías de resguardo de la identidad, acceso y fines de la información).

Por otra parte, cada organización firmó los consentimientos correspondientes a nivel institucional. Posterior a la aplicación, se ejecutó una depuración en base a un análisis factorial exploratorio con el programa IBM SPSS Statistics v.22, mediante mínimos cuadrados generalizados. Para establecer la validez de constructo de la escala se utilizó Análisis de Componentes Principales y Rotación Normalización Varimax. La consistencia interna y fiabilidad de la escala se estableció a través de alfa de Cronbach cuyos parámetros interpretativos consideraron valores entre 0,70 y 0.95 como aceptables (Terweea et al.,2007). 


\section{Fase 4: Análisis factorial confirmatorio}

Se aplicó la versión de 41 ítems a la submuestra B con el propósito de confirmar el modelo de medida establecido considerando en este proceso los mismos resguardos éticos explicitados en la Fase 3 . Posterior a la aplicación, se ejecutó un análisis factorial confirmatorio con el método de estimación mínimos cuadrados ponderados multivariantes a través del programa MPLUS v. 7.4. Las estimaciones de fiabilidad se establecieron en base al alfa de Cronbach. Para la interpretación de los datos se utilizaron los siguientes parámetros: Root Mean Square Error of Approximation (RMSEA) valores inferiores a 0.06 se consideran con ajuste óptimo y valores de 0.061 a 0.08 aceptables (Hu y Bentler, 1999). Para Comparative Fix Index (CFI) e Index Tucker-Lewis (TLI), valores entre 0.90 y 0.95 indican buen ajuste (Marsh et al., 2009).

\section{RESULTADOS}

A continuación, se presentan los resultados obtenidos de la fase 3 y 4 de la investigación, separados en resultados del análisis factorial exploratorio y resultados del análisis factorial confirmatorio

\section{Análisis Factorial Exploratorio}

Se realiza un examen inicial que arroja datos adecuados para las técnicas de análisis factorial exploratorio $(\mathrm{KMO}=0.78)$. El Análisis de Componentes Principales (Tabla 1), consolida las 5 dimensiones establecidas para los 41 ítems depurados.

Tabla 1: Análisis de Componentes Principales de la Escala de Autoeficacia en el Trabajo

\begin{tabular}{|c|c|c|c|c|c|}
\hline \multirow[b]{2}{*}{ Reactivo } & \multicolumn{5}{|c|}{ Factor } \\
\hline & Autogestión & Transferencia & $\begin{array}{c}\text { Meta- } \\
\text { cognición }\end{array}$ & $\begin{array}{c}\text { Meta- } \\
\text { aprendizaje }\end{array}$ & Autocuidado \\
\hline $\begin{array}{l}\text { 11. Para hacer bien algo, primero hay que } \\
\text { planificarlo. }\end{array}$ & 0.786 & & & & \\
\hline $\begin{array}{l}\text { 13. Siento que es importante planificar bien mí } \\
\text { tiempo en el trabajo. }\end{array}$ & 0.716 & & & & \\
\hline $\begin{array}{l}\text { 16. Utilizo estrategias que me permitan actuar } \\
\text { asertivamente. }\end{array}$ & 0.668 & & & & \\
\hline $\begin{array}{l}\text { 21. Elaborar estrategias que me permitan cumplir } \\
\text { con las metas en mi trabajo. }\end{array}$ & 0.648 & & & & \\
\hline 25. Expreso mis emociones asertivamente. & 0.617 & & & & \\
\hline 29. Me agrada planificar mi labor. & 0.569 & & & & \\
\hline $\begin{array}{l}\text { 32. Si controlo el desarrollo de mis labores, podré } \\
\text { mejorar mi trabajo. }\end{array}$ & 0.527 & & & & \\
\hline $\begin{array}{l}\text { 34. Uso herramientas que me permiten ser más } \\
\text { autónomo. }\end{array}$ & 0.521 & & & & \\
\hline $\begin{array}{l}\text { 36.Realizo actividades que fomenten } \mathrm{mi} \\
\text { autocuidado. }\end{array}$ & 0.495 & & & & \\
\hline 39. Autorregulo mis emociones. & 0.480 & & & & \\
\hline $\begin{array}{l}\text { 41. Es importante actuar asertivamente en el } \\
\text { contexto laboral. }\end{array}$ & 0.318 & & & & \\
\hline $\begin{array}{l}\text { 1. Considero valioso para mi persona llevar a la } \\
\text { acción un conocimiento adquirido. }\end{array}$ & & 0.652 & & & \\
\hline 5. Me agrada aprender a transferir aprendizajes. & & 0.600 & & & \\
\hline $\begin{array}{l}\text { 10. Ocupo estrategias que permiten mejorar mi } \\
\text { trabajo. }\end{array}$ & & 0.561 & & & \\
\hline $\begin{array}{l}\text { 12. Utilizo estrategias que me ayudan a } \\
\text { adaptarme a las exigencias formales en mi } \\
\text { trabajo. }\end{array}$ & & 0.510 & & & \\
\hline 17. Me agrada realizar un buen trabajo. & & 0.482 & & & \\
\hline $\begin{array}{l}\text { 19. Siento que soy capaz de aprender a } \\
\text { conocerme mejor. }\end{array}$ & & 0.433 & & & \\
\hline $\begin{array}{l}\text { 20. Conozco mis fortalezas, oportunidades, } \\
\text { debilidades y amenazas en mi trabajo. }\end{array}$ & & 0.394 & & & \\
\hline $\begin{array}{l}\text { 23. Conozco perfectamente el rol que desempeño } \\
\text { en mi lugar de trabajo. }\end{array}$ & & 0.384 & & & \\
\hline $\begin{array}{l}\text { 26. Cuando adquiero nuevos conocimientos los } \\
\text { comparto con mis compañeros. }\end{array}$ & & 0.380 & & & \\
\hline
\end{tabular}


Tabla 1: continuación.

\begin{tabular}{|c|c|c|c|c|c|}
\hline Reactivo & Autogestión & Transferencia & $\begin{array}{c}\text { Meta- } \\
\text { cognición }\end{array}$ & $\begin{array}{c}\text { Meta- } \\
\text { aprendizaje }\end{array}$ & Autocuidado \\
\hline $\begin{array}{l}\text { 3. Planifico las tareas de mi trabajo } \\
\text { adecuadamente. }\end{array}$ & & & 0.741 & & \\
\hline $\begin{array}{l}\text { 6. Realizo un buen trabajo gracias a que } \\
\text { monitoreo mi forma de trabajar. }\end{array}$ & & & 0.632 & & \\
\hline $\begin{array}{l}\text { 9. Creo que es importante respetar normas en } \\
\text { el trabajo. }\end{array}$ & & & 0.578 & & \\
\hline $\begin{array}{l}\text { 15. Mantengo una convivencia agradable con } \\
\text { mis compañeros de trabajo. }\end{array}$ & & & 0.516 & & \\
\hline $\begin{array}{l}\text { 18. Siempre realizo un seguimiento de } \mathrm{mi} \\
\text { trabajo }\end{array}$ & & & 0.441 & & \\
\hline $\begin{array}{l}\text { 22. Presto atención a los errores que cometo } \\
\text { para no volver a repetirlos. }\end{array}$ & & & 0.420 & & \\
\hline $\begin{array}{l}\text { 24. Reconozco las emociones que perjudican } \\
\text { mi desarrollo laboral. }\end{array}$ & & & 0.379 & & \\
\hline $\begin{array}{l}\text { 28. Algunas dificultades que surgen en el } \\
\text { trabajo las supero gracias a mi } \\
\text { autoconocimiento. }\end{array}$ & & & 0.375 & & \\
\hline $\begin{array}{l}\text { 30. Me desagrada saber que en mi trabajo no } \\
\text { se ocupan eficazmente los recursos. }\end{array}$ & & & 0.313 & & \\
\hline $\begin{array}{l}\text { 2. Me alegra saber que puedo conocer mi } \\
\text { propia manera de aprender. }\end{array}$ & & & & 0.920 & \\
\hline $\begin{array}{l}\text { 7. Pongo atención cuando me hablan sobre } \\
\text { cómo mejorar mi aprendizaje. }\end{array}$ & & & & 0.801 & \\
\hline $\begin{array}{l}14 \text { Siento que si conozco como aprendo, podré } \\
\text { aprovechar mejor la información. }\end{array}$ & & & & 0.717 & \\
\hline $\begin{array}{l}\text { 37. Siento que soy capaz de aprender cosas } \\
\text { nuevas. }\end{array}$ & & & & 0.625 & \\
\hline $\begin{array}{l}\text { 40. Me intereso en conocer y mejorar mi propio } \\
\text { estilo de aprendizaje. }\end{array}$ & & & & 0.471 & \\
\hline $\begin{array}{l}\text { 4. El autocontrol es necesario para las } \\
\text { relaciones interpersonales. }\end{array}$ & & & & & 0.789 \\
\hline 8. Soy feliz cuando todo sale como lo planeo. & & & & & 0.703 \\
\hline $\begin{array}{l}\text { 27. Estoy seguro(a) que puedo mejorar mi } \\
\text { capacidad de aplicar nuevos conocimientos. }\end{array}$ & & & & & 0.491 \\
\hline $\begin{array}{l}\text { 31. Me preocupo por mi higiene y apariencia } \\
\text { personal. }\end{array}$ & & & & & 0.484 \\
\hline 33. Trato de mejorar mi forma de hablar. & & & & & 0.456 \\
\hline $\begin{array}{l}\text { 35. Conocerme me sirve para trabajar de } \\
\text { manera más eficaz }\end{array}$ & & & & & 0.398 \\
\hline $\begin{array}{l}\text { 38. Para mí es importante cuidarme en el } \\
\text { trabajo. }\end{array}$ & & & & & 0.389 \\
\hline Porcentaje varianza total explicada & $13.2 \%$ & $12.88 \%$ & $9.42 \%$ & $8.78 \%$ & $7.91 \%$ \\
\hline
\end{tabular}

El total de varianza explicada por los 5 componentes principales de la Escala de Autoeficacia en el Trabajo es de $52.1 \%$. En la Tabla 2 se encuentran los alfas de Cronbach de cada una de las dimensiones. El análisis factorial exploratorio arroja un valor de alfa de Cronbach para la escala total de 0.931 lo que revela un adecuado índice de consistencia interna.

Tabla 2: Consistencia Interna Escala de Autoeficacia en el Trabajo

\begin{tabular}{|l|c|c|}
\hline Dimensiones & $N^{\circ}$ de ítems & alfa de Cronbach \\
\hline Autogestión & 11 & 0.878 \\
\hline Transferencia & 9 & 0.819 \\
\hline Metacognición & 9 & 0.875 \\
\hline Meta-Aprendizaje & 5 & 0.759 \\
\hline Autocuidado & 7 & 0.780 \\
\hline
\end{tabular}




\section{Análisis Factorial Confirmatorio}

Con el propósito de confirmar el modelo de medida obtenido se aplicó la escala de autoeficacia en el trabajo a la submuestra B con el objetivo de ejecutar un análisis factorial confirmatorio (AFC). Los indicadores de ajuste del modelo (Tabla 3 ) arrojan niveles aceptables a partir de los parámetros señalados en la literatura especializada, lo cual demuestra que la escala cuenta con estabilidad y mantiene su estructura factorial.

Tabla 3: Indicadores de Ajuste Escala de Autoeficacia en el Trabajo

\begin{tabular}{|c|c|}
\hline Índice de Ajuste & $\begin{array}{c}\text { Modelo } \\
\text { Autoeficacia en el trabajo }\end{array}$ \\
\hline CFI & 0.97 (buen ajuste) \\
\hline TLI & 0.97 (buen ajuste) \\
\hline RMSEA & 0.05 (ajuste óptimo) \\
\hline
\end{tabular}

La Figura 1 y Figura 2 dan cuenta de las estimaciones de saturaciones factoriales a partir del modelo extraído. Los 41 reactivos finales arrojan representaciones aceptables $(\lambda \geq 0.50)$ o excelentes $(\lambda \geq 0.70)$ de las variables latentes (Hair et al., 2014) Se certifican las 5 dimensiones establecidas previamente: autocuidado (7 ítems), meta-aprendizaje (5 ítems), meta-cognición (9 ítems), transferencia (9 ítems) y autogestión (11 ítems).

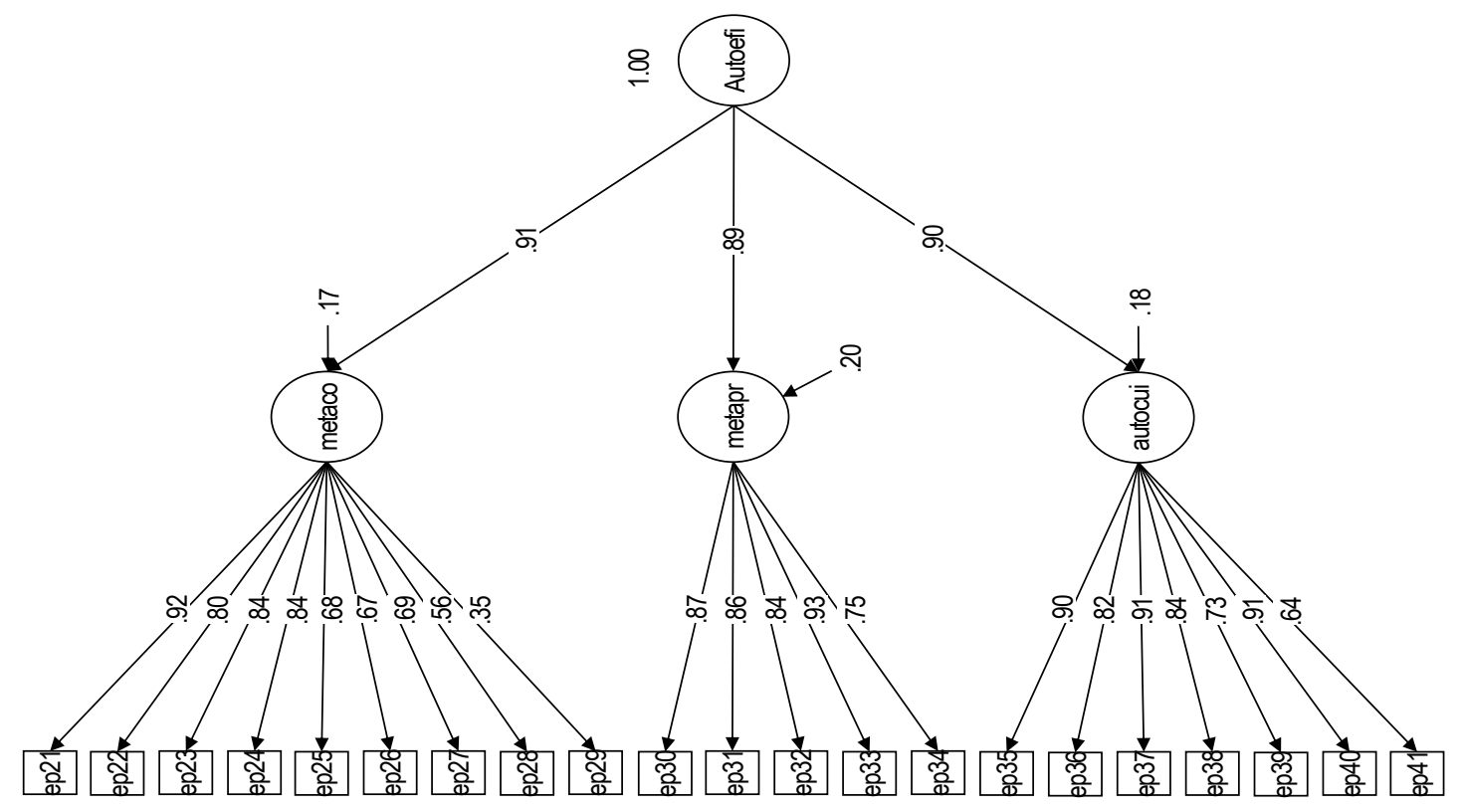

Fig. 1. Estructura Factorial Escala de Autoeficacia en el Trabajo

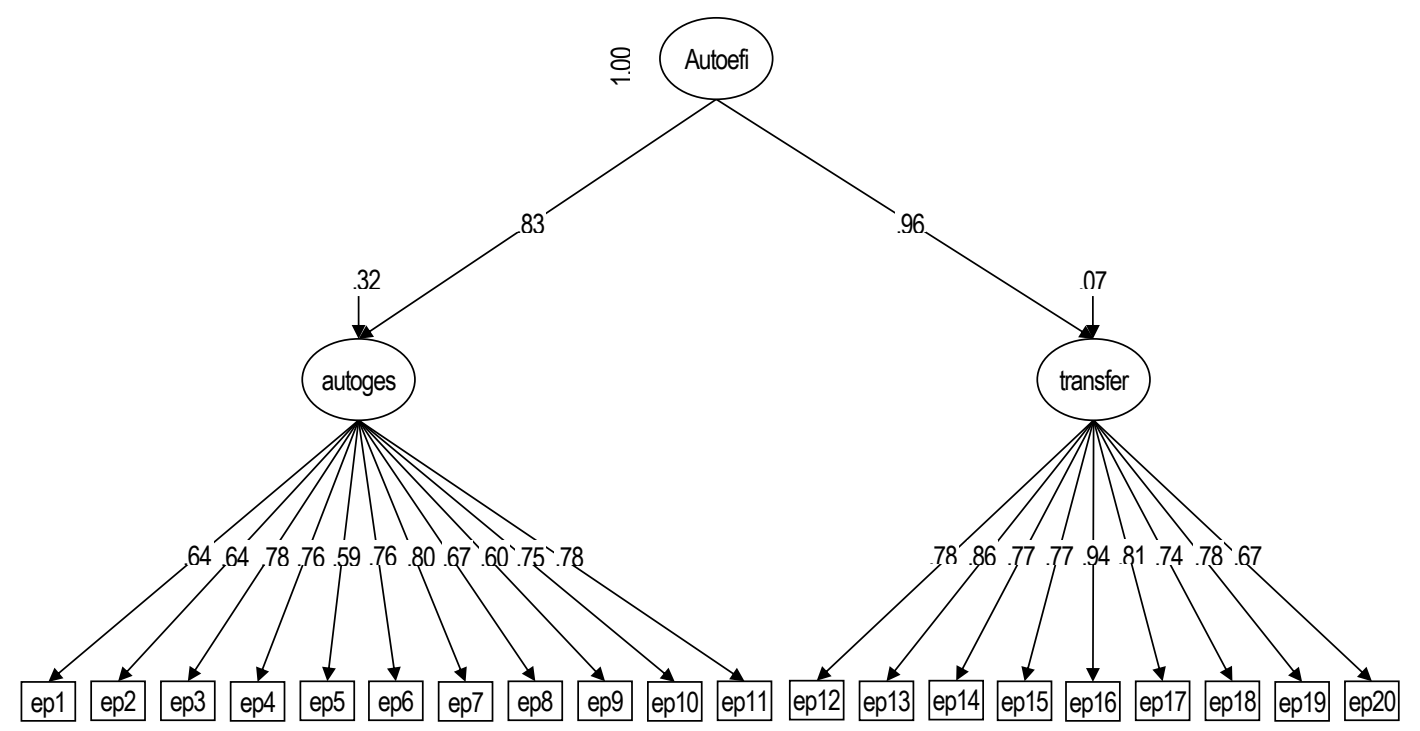

Fig. 2. Estructura Factorial Escala de Autoeficacia en el Trabajo 
El análisis factorial confirmatorio arroja un alfa de Cronbach de 0.948 para la escala total de autoeficacia en el trabajo, lo que demuestra una adecuada consistencia interna al igual que cada una de sus dimensiones por separado (Tabla 4). La escala de 41 ítems construida en formato Likert cuenta con 4 categorías de respuesta (totalmente en desacuerdo, en desacuerdo, de acuerdo, totalmente de acuerdo), calificados de 1 (totalmente en desacuerdo) a 4 (totalmente de acuerdo). La puntuación total indica el nivel de autoeficacia en el trabajo en base a un sistema de clasificación cuya suma por dimensiones permite obtener un registro ordinal.

Tabla 4: Coeficientes de Fiabilidad

\begin{tabular}{|c|c|c|c|c|c|}
\cline { 2 - 6 } \multicolumn{1}{c|}{} & \multicolumn{5}{c|}{ Dimensión } \\
\hline Estadístico & Autogestión & Transferencia & Meta-cognición & Meta-aprendizaje & Autocuidado \\
\hline alfa de Cronbach & 0.858 & 0.869 & 0.808 & 0.826 & 0.854 \\
\hline
\end{tabular}

Para establecer los puntajes de la escala se utilizaron las puntuaciones típicas o estándar lineales directas, las que señalan la distancia, en términos de desviaciones típicas, a que se encuentra un puntaje original con respecto a la media de su distribución de puntajes originales. En la submuestra $B$ la media corresponde a $(X=146.06)$ y desviación Estándar $(\sigma=12.95)$. En esta muestra los trabajadores que alcanzan puntajes inferiores a 133,11 poseen baja autoeficacia en el trabajo; entre 159.01 y 133.11 corresponden a autoeficacia media y puntajes superiores a 159.01 califican alta autoeficacia en el trabajo.

\section{DISCUSIÓN}

De acuerdo a los resultados obtenidos se puede afirmar que la Escala de Autoeficacia en el Trabajo (EAT) compuesta por 41 reactivos de tipo diferencial semántico distribuidos en cinco dimensiones: autocuidado, meta-aprendizaje, metacognición, transferencia y autogestión, cuenta con las características psicométricas requeridas para medir el constructo en estudio, aportando una herramienta de calidad a organizaciones, trabajadores e investigadores, por cuanto contribuye a los procesos de diagnóstico y evaluación (Tabla 5).

Las dimensiones que componen la escala de autoeficacia en el trabajo coinciden con las investigaciones realizadas por diversos autores. En el caso de autogestión se encuentra lo planteado por Minjire et al. (2020) quienes encontraron una correlación positiva de fuerza moderada entre autogestión y autoeficacia, lo que destaca la relevancia de ejercitar el control o agencia personal sobre el propio funcionamiento (Bandura, 1989; Sora et al., 2019).

Respecto a la dimensión de autocuidado coincide con los resultados obtenidos por Kind et al., (2020) los cuales demuestran una correlación alta positiva entre autocuidado y autoeficacia, lo que realza la importancia de esta dimensión para alcanzar la salud psicológica, emocional y física aumentando la capacidad de resiliencia de los sujetos frente a los eventos que afectan su vida y desempeño laboral (Kwong y Kwan, 2007; Park et al., 2020). Por otra parte, Na-Nan y Sanamthong (2019) plantean a partir de sus resultados que la transferencia de motivación, entrenamiento, conocimiento y habilidades constituye una variable mediadora de la autoeficacia.

Por su parte, Lyons y Bandura (2019) demuestran que las variables de autoeficacia y metacognición están altamente relacionadas entre sí e impactan en el desempeño en el trabajo, lo que devela la relevancia de los niveles de conciencia del sujeto acerca de sus debilidades y fortalezas para enfrentar los desafíos en el contexto laboral (Siriphat y Wedchayanon, 2017). Finalmente, Colthorpe et al. (2019) establecen la relación positiva entre metacognición, meta-aprendizaje y autoeficacia, entre otras variables y concluyen que la metacognición y el meta-aprendizaje favorecen el involucramiento del sujeto con estrategias más diversas de aprendizaje, principalmente aquellas relacionadas a las fases de previsión y autorreflexión aumentando sus niveles de conciencia (Colthorpe et al., 2019). Asimismo, Olaniyi (2020) confirma la relación positiva entre meta aprendizaje y autoeficacia lo que significa que las mejoras en el proceso de meta-aprendizaje se relacionan con mejoras en los niveles de autoeficacia.

La escala de Autoeficacia en el Trabajo permite diseñar intervenciones focalizadas que potencien el desempeño laboral exitoso y trabajadores con mayor bienestar (Brown et al., 2008; Sora et al., 2019). Quienes confían en sus propias capacidades tienden a percibir las dificultades laborales como menos amenazantes y más como desafíos, por lo cual experimentan resultados menos perjudiciales en el logro de sus metas que aquellos que presentan baja autoeficacia en el trabajo (Sora et al., 2019). 
Tabla 5: Escala de Autoeficacia en el Trabajo

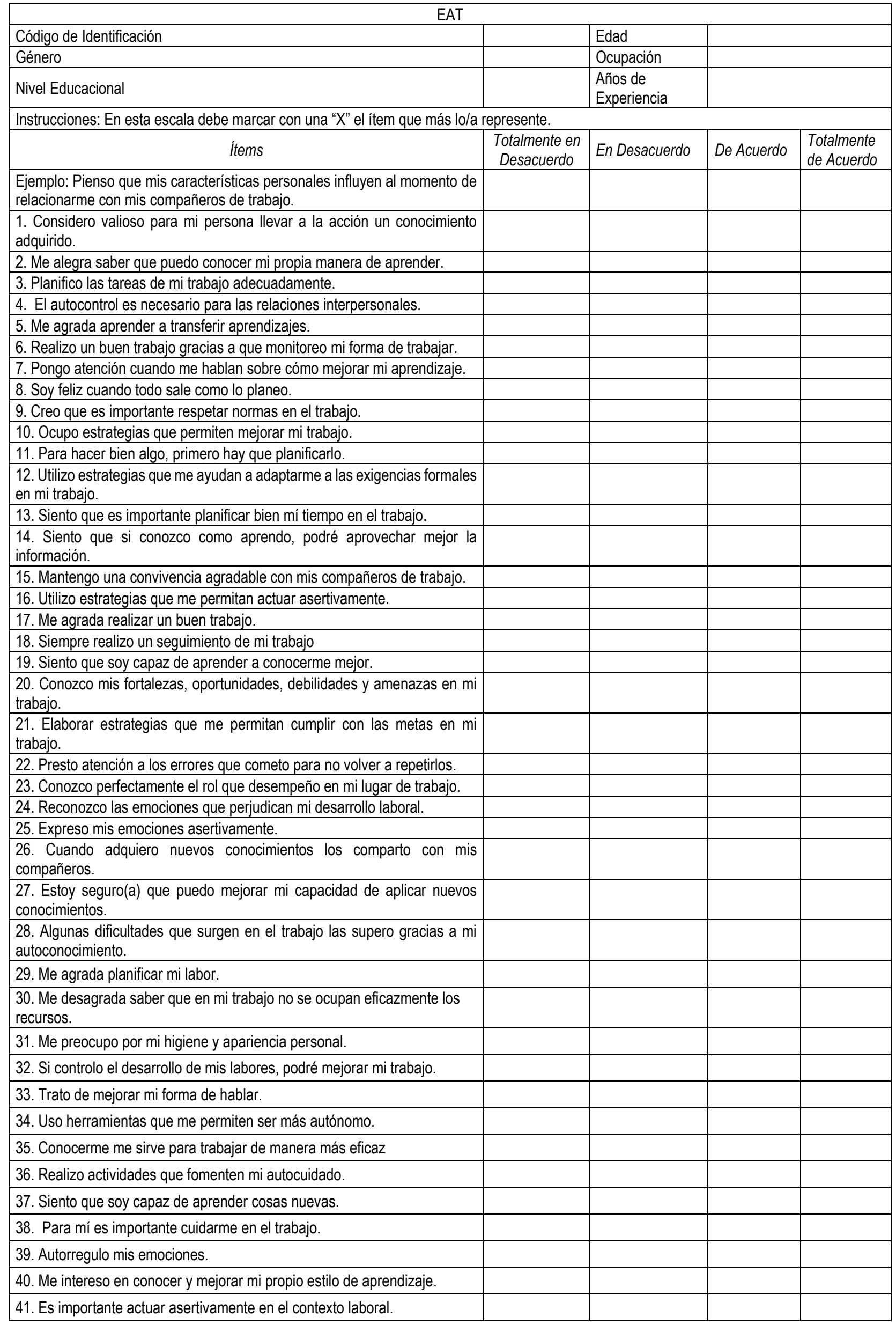




\section{CONCLUSIONES}

El propósito de este estudio fue construir y validar una Escala de Autoeficacia en el Trabajo para contextos laborales transversales. Tanto la escala total como cada una de sus dimensiones: Autogestión, Transferencia, Metacognición, Meta-Aprendizaje y Autocuidado poseen indicadores psicométricos sólidos permitiendo su uso de manera independiente, aplicable a cualquier ámbito laboral. Los resultados obtenidos permiten afirmar que la escala creada (EAT) constituye un significativo aporte a las demandas diagnósticas de la Psicología del Trabajo y las Organizaciones, tanto a nivel de investigación como de acción/intervención.

\section{REFERENCIAS}

Bargsted, M., Ramírez-Vielma, R., y Yeves, J., Professional self-efficacy and job satisfaction: The mediator role of work design, https://doi.org/10.5093/jwop2019a18, Journal of Work and Organizational Psychology, 35, 157-16 (2019)

Bandura, A., Human agency in social cognitive theory, https://doi.org/10.1037/0003-066X.44.9.1175, American Psychologist, 44(9), 1175-1184 (1989)

Bandura, A., Self-efficacy: The exercise of control, Freeman, New York, USA (1997)

Brown, S.D., Tramayne, S., y otros cuatro autores, Social cognitive predictors of college students academic performance and persistence: A meta-analytic path analysis, https://doi.org/10.1016/j.jvb.2007.09.003, Journal of Vocational Behavior, 72(3), 298-308 (2008)

Calderón, J.L., Laca, F., y Pando, M., La autoeficacia como mediador entre el estrés laboral y el bienestar, http://dx.doi.org/10.5231/psy.writ.2017.1511, Psicología y Salud, 27(1), 71-78 (2017)

Cancio-Bello, C., Lorenzo, A., y Alarcó, G., Autocuidado: Una aproximación teórica al concepto, http://dx.doi.org/10.18566/infpsic.v20n2a9, Informes Psicológicos, 20(2), 119-138 (2020)

Cerda, C., López, O., Osses, S., y Saiz, J.L., Análisis psicométrico de la escala de aprendizaje de autodirigido basada en la teoría de aprendizaje autodirigido de garrison, Revista Iberoamericana de Diagnóstico y Evaluación - e Avaliação Psicológica, 1(39), 46-56 (2015)

Cid, P., Orellana, A., y Barriga, O., Validación de la escala de autoeficacia general en Chile, http://dx.doi.org/10.4067/S0034-98872010000500004, Revista Médica de Chile, 138(5), $551-557$ (2010)

Colthorpe, K., Ogiji, J., y otros tres autores, Effect of metacognitive pormpts on undergraduate pharmacy students' selfregulated learning baheavior, DOI:10.5688/ajpe6646, American Journal of Pharmaceutical Education, 83(4), 536-536 (2019)

Criollo, M., Romero, M., y Fontaines-Ruiz, T., Autoeficacia para el aprendizaje de la investigación en estudiantes universitarios, https://doi.org/10.1016/j.pse.2016.09.002, Psicología Educativa, 23, 63-72 (2017)

Gonczy, A., Análisis de las tendencias internacionales y de los avances en educación y capacitación laboral basadas en normas de competencias, Argüelles, A., y Gonczy, A. Educación y capacitación basadas en normas de competencias, 3840, Limusa, México (2001)

Hair, J., Black, W., Babin, B., y Anderson, R., Multivariate data analysis, $7^{\circ}$ ed., Pearson Education Limited, England (2014)

$\mathrm{Hu}$, L. y Bentler, P.M. Cutoff criteria for fit indexes in covariance structure analysis: conventional criteria versus new alternatives, DOI: 10.1080/10705519909540118, Structural Equation Modeling: A Multidisciplinary Journal, 6(1), 1-55 (1999)

Kind, N., Bürgin, D., Fegert, J.M., y Schmid, M., What protects youth residential caregivers from burning out? a longitudinal analysis of individual resilience, DOI:10.3390/ijerph17072212, International Journal of Environmental Research and Public Health, 17(2212), 1-12 (2020)

Kwong, E.W., y Kwan, A.Y., Participation in health promoting behaviour: Influences on community-dwelling older chinese people, https://doi.org/10.1111/j.1365-2648.2006.04132.x, Journal of Advanced Nursing, 57(5), 522-534 (2007)

Lyons, P.R., y Bandura, R.P., Exploring linkages of performance with metacognition, http://dx.doi.org/10.1108/JMD-072018-0192, The Journal of Management Development, 38(3), 195-207 (2019)

Marsh, H.W., Muthén, B., y otros 4 autores, Exploratory structural equation modeling, integrating CFA and EFA: Application to students' evaluations of university teaching, https://doi.org/10.1080/10705510903008220, Structural Equation Modeling: A Multidisciplinary Journal, 16, 439-576 (2009)

McCluskey, K., Treffinger, D., Baker, P., y Lamoureux, K., The amphitheater model for talent development: recognizing and nurturing the gifts of our lost prizes, International Journal for Talent Development and Creativity, 1(1), 99-112 (2013)

Montero, I., y León, O., A guide for naming research studies in psychology, International Journal of Clinical and Health Psychology, 7(3), 847-862 (2007)

Minjire, N., Gachunga, H., y Mwanki, J., Influence of principals' self-management on the organisational commitment of teachers in secondary school in county governments in Kenya, DOI: 10.14807/ijmp.v11i4.1031, Independent journal of management \& production, 11(4), 434-1478 (2020) 
Na-Nan, K., y Sanamthong, E., Self-efficacy and employee job performance, http://dx.doi.org/10.1108/IJQRM-01-20190013, The International Journal of Quality \& Reliability Management, 36(1), 1-17 (2019)

Olaniyi Nkaepe, E.E., Threshold concepts: designing a format for the flipped classroom as an active learning technique for crossing the threshold, http://dx.doi.org/10.1186/s41039-020-0122-3, Research and Practice in Technology Enhanced Learning, 15(2), 1-15 (2020)

Park, M., Song, S., y Kim, J., The mediating effect of childcare teachers's resilience on the relationship between social support in the workplace and their self-care, DOI:10.3390/ijerph17228513, International Journal of Environmental Research and Public Health, 17(8513), 1-15 (2020)

Pepe, S.J., Garnese, M.L., Avalone, F., y Vecchioe, M., Work self-efficacy scale and search for work self-efficacy scale: a validation study in spanish and italian cultural contexts, DOI: 10.5093/tr2010v26n3a4, Revista de Psicología del Trabajo y de las Organizaciones, 26(3), 201-210 (2010)

Ramos-Villagrasa, P.J., Sánchez-Iglesias, I., y otros cuatro autores, Spanish version of "self-efficacy for writing scale" (SEWS), http://dx.doi.org/10.6018/analesps.34.1.264931, Annals of Psychology, 53(1), 86-91 (2018)

Siriphat, P. y Wedchayanon, N., An empirical study of the relationship between job demand-control-support model, selfefficacy, burnout, informal learning, and job performance: a case of employees in the thai banking sector, AU-GSB EJournal, 10(1),195-203 (2017)

Sora, B., Höge, T., Caballer, A., y Peiró, J.M., Employment contract, job insecurity and employees' affective well-being: the role of self- and collective efficacy, DOI: 10.1177/0143831X18804659, Economic and Industrial Democracy, 40(2), 193-214 (2019)

Terweea, C., Bot, S.D.M., y otros 4 autores, Quality criteria were proposed for measurement properties of health status questionnaires, https://doi.org/10.1016/j.jclinepi.2006.03.012, Revista de epidemiología clínica, 60(1), 34-42 (2007)

Updegraff, S., Maximizing human potential: Tips to foster personal effectiveness, DOI: 10.1002/ert.20050, Employment Relations Today, 31(1), 43-50 (2004)

Vera, M., Salanova, M., y Martín del Río, B., Self-efficacy among university faculty: how to develop an adjusted scale, Anales de Psicología, 27(3), 800-807 (2011) 